\title{
Cáncer de Colon y Recto: Descripción Morfológica y Clínica de 322 Casos
}

\author{
Colon and Rectum Cancer: Morphological and Clinical Description of 322 Cases
}

\author{
"Oscar Tapia E.; *Juan Carlos Roa S.; "* Carlos Manterola D. \& "Enrique Bellolio J.
}

TAPIA, E. O.; ROA, S. J. C.; MANTEROLA, D. C. \& BELLOLIO, J. E. Cáncer de colon y recto: descripción morfológica y clínica de 322 casos. Int. J. Morphol., 28(2):393-398, 2010.

RESUMEN: El cáncer de colon y recto (CCR) es actualmente la cuarta causa de muerte por cáncer en Chile. Su incidencia, sin embargo, está aumentando continuamente en nuestra población. El objetivo de este estudio es describir aspectos morfológicos y clínicos de pacientes resecados por CCR. Estudio de cohorte retrospectiva. Se estudiaron 322 pacientes intervenidos por CCR entre 1987 y 2003 en el Hospital Hernán Henríquez Aravena de Temuco. Las variables clínicas y morfológicas estudiadas (todas ellas analizadas para los subgrupos de sujetos con tumores de colon y de recto) fueron edad, género, localización tumoral, forma y tamaño tumoral, nivel de infiltración, tipo histológico, grado de diferenciación histológico y compromiso tumoral de nodos linfáticos. Se utilizaron estadísticas descriptivas y analíticas; aplicando chi-cuadrado de Pearson y exacto de Fisher para las variables categóricas; y, T-test para variables continuas. La mediana de edad fue de 66 años, con promedio de edad para tumores de colon derecho (CD), transverso (CT), izquierdo (CI) y recto fue $62,2,64,6,64$, y 64,4 años respectivamente $(\mathrm{p}=0,53)$. En CD e CI se verificaron $57 \%$ y $47 \%$ de mujeres respectivamente (p<0,05). El 69\% de los casos correspondió a tumores de colon $(24 \%$ CD, $4 \%$ CT y $41 \%$ CI) y $31 \%$ a tumores de recto. El tamaño tumoral promedio fue $67,2 \pm 33,1 \mathrm{~mm} \mathrm{CD}, 53,5 \pm 19,7 \mathrm{~mm} \mathrm{CT}, 44,1 \pm 22,3 \mathrm{~mm}$. CI y 41,5 $\pm 17,5 \mathrm{~mm}$ en recto (p<0,001). En CD la forma tipo Bormann I se observó en el $57 \%$ mientras que en CI lesiones anulares y ulceradas en $45 \%$ en CI. Se encontró $75 \%$ de tumores moderadamente diferenciados; correspondiendo el $82 \%$ a adenocarcinomas, $16 \%$ adenocarcinoma mucinoso y $2 \%$ carcinoma de células en anillo de sello. El 76\% correspondió a tumores T3 y T4. Se encontró compromiso tumoral de nodos linfáticos en $39 \%$ de los cuales el 95\% correspondían a tumores T3-T4. Se verificaron a nivel regional para pacientes con CCR las variables clínicas y morfológicas descritas en la literatura.

PALABRAS CLAVE: Cancer colorectal; Cáncer de colon; Cáncer de recto.

\section{INTRODUCCIÓN}

El cáncer colorrectal $(\mathrm{CCR})$ corresponde a todos aquello tumores ubicados en el intestino grueso, pudiendo estos tumores localizarse por lo tanto desde la valva ileocecal hasta el recto. En el mundo, cada año se registran aproximadamente un millón de nuevos casos de cáncer colorrectal (CCR) y medio millón de muertes, con una tasa cruda mundial de mortalidad de 8,1/100.000 hab.; las que afectan principalmente a las regiones más desarrolladas $(25,1 / 100.000$ hab.), mientras que en las de menor desarrollo la tasa es significativamente inferior (3,9/100.000 hab.). (Bannura et al., 1995; Bannura, 2006). En Chile, se ha observado un aumento en la incidencia y mortalidad del CCR en los últimos años, ocupando actualmente la cuarta causa de muerte por cáncer, con una tasa de mortalidad general de 6,4 x 105 hab (Donoso et al., 2006; Landis et al., 1999; López et al., 2003; Bannura).

Si bien estos tumores pueden localizarse desde la valva ileocecal al recto, algunos trabajos han intentado agruparlos de acuerdo a su localización en tumores proximales o derechos y distales o izquierdos según su relación con el ángulo izquierdo del colon teniendo como fundamento para ello el diferente origen embriológico de estos segmentos; derivando el colon proximal del intestino primitivo medio e irrigado por la arteria mesentérica superior mientras que el colon distal deriva del intestino primitivo posterior y es irriga-

\footnotetext{
* Departamento de Anatomía Patológica. Facultad de Medicina. Universidad de La Frontera. Temuco, Chile.

** Departamento de Cirugía y Traumatología, Facultad de Medicina. Universidad de La Frontera. Temuco, Chile.

Financiado en parte por la Dirección de investigación de la Universidad de La Frontera. DIUFRO 120528.
} 
do por la arteria mesentérica inferior; esta clasificación, sin embargo, carece de un valor pronóstico y actualmente estos son agrupados en tumores de colon derecho, transverso, izquierdo y recto (Parkin et al., 1992; Ziegler et al., 1986). Un punto importante, es la dificultad que existe para definir la ubicación tumoral a nivel de rectosigmoides, pues no existe una clara delimitación anatómica que permita con seguridad determinar la localización, aceptándose convencionalmente hoy en día como recto a los $15 \mathrm{~cm}$ proximales desde el margen anal. La importancia en definir si el cáncer es de colon o recto radica en el distinto comportamiento pronóstico descrito en numerosas series; diferencias que se explicarían por la mayor tasa de recidiva local de los tumores de recto; condición determinada entre otros factores por el estadio tumoral, localización y experiencia del equipo quirúrgico (López et al.; Bannura et al., 1995; Parkin et al.; Ziegler et al.; Bannura et al., 2001).

Además de la localización, el análisis de la pieza operatoria permite identificar otras variables morfológicas de estos tumores como es la forma tumoral; se han descrito lesiones anulares y estenosantes más frecuentemente en el colon izquierdo a diferencia de los tumores polipoídeos observados en el colon derecho. Así también el mayor tamaño descrito para cánceres de localización cecal, producto de su crecimiento exofítico, siendo ellos generalmente diagnosticados en estadios avanzados y en ocasiones pesquisados clínicamente por la presencia de una masa palpable en fosa iliaca derecha durante el examen abdominal; por el contrario tumores de colon izquierdo al ser lesiones estenosantes dan sintomatología obstructiva intestinal lo que motiva su estudio y en un porcentaje no menor su primera manifestación corresponde a una obstrucción intestinal (Speights et al., 1991; Barillari et al., 1989; Qizilbash et al., 1982; Fenoglio, 2008; Odze \& Goldblum, 2009).

Desde el punto de vista histológico, la Organización Mundial de la Salud considera las siguientes variantes histológicas para carcinomas de colon y recto: adenocarcinoma, adenocarcinoma mucinoso, carcinoma de células en anillo de sello, carcinoma de células pequeñas, carcinoma medular, carcinoma indiferenciado y carcinoma neuroendocrino; siendo más frecuente el adenocarcinoma y clasificándose estos a su vez de acuerdo al grado de diferenciación en tumores bien, moderado o poco diferenciados (Fenoglio, Odze \& Goldblum; WHO, 2000).

Un elemento morfológico pronóstico importante en este grupo de pacientes es el nivel de infiltración tumoral en la pared intestinal, utilizándose para ello el estadio propuesto por la AJCC, el cual considera para su estadificación el nivel de invasión tumoral ya sea en túnica mucosa, submucosa, muscular propia, subserosa o serosa. Cabe mencionar la ausencia de túnica serosa a nivel rectal, por lo que estos tumores infiltran directamente en el tejido adiposo perirrectal o adventicial (AJCC, 2002).

En base a lo anteriormente expuesto, el objetivo de este estudio es describir aspectos morfológicos y clínicos de pacientes resecados por CCR en el Hospital Hernán Henríquez Aravena de Temuco entre Enero de 1993 y Diciembre de 2003.

\section{MATERIAL Y MÉTODO}

\section{Diseño del estudio: Cohorte retrospectiva.}

Marco: El estudio fue realizado en el Departamento de Anatomía Patológica de la Universidad de La Frontera, donde se recogieron los datos generados entre enero de 1993 y diciembre de 2003. Se utilizó un muestreo no probabilístico de casos consecutivos.

Participantes: Se incluyeron pacientes que fueron resecados por CCR de forma electiva en el Hospital Hernán Henríquez Aravena de Temuco-Chile, en el período antes señalado ( $\mathrm{n}=$ 322). Los sujetos incluidos en el estudio fueron confirmados en su diagnóstico mediante la revisión dirigida de las piezas quirúrgicas y láminas histológicas. La totalidad de los pacientes incluidos finalmente en el estudio fueron intervenidos quirúrgicamente por un equipo de cirujanos digestivos con formación en el área y de acuerdo a protocolos establecidos para el tratamiento del cáncer de colon y recto.

Variables de interés: Se estudiaron variables clínicas (edad y género) obtenidas a partir de los registros de las historias clínicas de cada sujeto; y morfológicas (localización tumoral, tamaño y forma tumoral, grado de diferenciación histológica, nivel de infiltración, tipo histológico y compromiso de nodos linfáticos), obtenidas a partir del estudio de las piezas quirúrgicas y láminas histológicas.

Medidas: Algunas de las siguientes variables en estudio fueron categorizadas: Edad, género, tamaño tumoral (medida en $\mathrm{mm}$ ), localización tumoral (colon derecho, transverso, izquierdo y recto), forma tumoral (poliposo, discoidal y anular- ulcerado), tipo histológico de acuerdo a la clasificación de la OMS (IARC), grado de diferenciación (bien, moderado y poco diferenciado), nivel de infiltración tumoral de acuerdo a la clasificación TNM propuesta por la AJCC para el cáncer de colon y recto (T1, T2, T3 y T4) (AJCC 2002), número de linfonodos resecados y número de linfonodos comprometidos por tumor. En relación a la localización tumoral se diferenciaron los tumores de colon y recto según los reparos anatómicos convencionalmente aceptados; defi- 
niendo la localización rectal a aquellos tumores ubicados en los $15 \mathrm{~cm}$ proximales al margen anal. Se identificaron tres formas tumorales: poliposo (Bormann I), discoidal (Bormann II) y anular-ulcerado (Bormann III).

Métodos estadísticos: Se utilizó estadística descriptiva con cálculo de medidas de tendencia central y extrema; y analítica, aplicando análisis divariados: T-student para variables continuas, chi cuadrado de Pearson y exacto de Fisher para variables categóricas; con un intervalo de confianza de $95 \%$ (IC 95\%).

\section{RESULTADOS}

La mediana de edad de la cohorte fue de 66 años ( 25 a 99 años). El promedio de edad para tumores de CD, CT, CI y recto fue 62,2 años, 64,6 años, 64 años y 64,4 años respectivamente $(\mathrm{p}=0,53)$. Se verificaron en el grupo estudiado $50 \%$ de mujeres, en tumores de $\mathrm{CD}$ el $57 \%$ correspondió a mujeres y en $\mathrm{CI}$ solo el $47 \%(\mathrm{p}<0,05)$.

El 69\% de los casos correspondió a tumores de colon $(24 \% \mathrm{CD}, 4 \% \mathrm{CT}$ y $41 \% \mathrm{CI})$ y el $31 \%$ restante a tumores de recto. El tamaño tumoral promedio de acuerdo a la localización fue $67,2 \pm 33,1 \mathrm{~mm}$ en $\mathrm{CD}, 53,5 \pm 19,7 \mathrm{~mm}$ en CT, $44,1 \pm 22,3 \mathrm{~mm}$ en colon izquierdo y $41,5 \pm 17,5 \mathrm{~mm}$ en recto $(\mathrm{p}<0,001)$.

La forma tumoral poliposa o tipo Bormann I (Fig. 1) correspondió al 57\% de los tumores ubicados en el CD mientras que lesiones anulares y ulceradas (tipo Bormann III) (Fig. 2) se observan en el $45 \%$ de los tumores del CI.

De las características histológicas analizadas se encontró un $75 \%$ de tumores moderadamente diferenciados; correspondiendo el $82 \%$ a adenocarcinomas de los cuales

Tabla I. Distribución de variables clínicas y morfológicas.

\begin{tabular}{|c|c|c|c|c|}
\hline & Colon Derecho & Colon Transverso & Colon Izquierdo & Recto \\
\hline & $24 \%(77)$ & $4 \%(13)$ & $41 \%(132)$ & $31 \%(100)$ \\
\hline Edad (promedio) & $62,2 \pm 12,3$ años & $64,6 \pm 16,6$ años & $64 \pm 14,2$ años & $64,4 \pm 12,7$ años \\
\hline \multicolumn{5}{|l|}{ Sexo } \\
\hline Femenino & $57 \%(44)$ & $69 \%(9)$ & $47 \%(62)$ & $48 \%(48)$ \\
\hline Masculino & $43 \%(33)$ & $31 \%(4)$ & $53 \%(70)$ & $52 \%(52)$ \\
\hline Tamaño tumoral (promedio) & $67,2 \pm 33,1 \mathrm{~mm}$ & $53,5 \pm 19,7 \mathrm{~mm}$ & $44,1 \pm 22,3 \mathrm{~mm}$ & $41,5 \pm 17,5 \mathrm{~mm}$ \\
\hline \multicolumn{5}{|l|}{ Forma } \\
\hline Poliposo & $57 \%(44)$ & - & $18 \%(24)$ & $14 \%(14)$ \\
\hline Ulcerado & $30 \%(23)$ & $54 \%(7)$ & $45 \%(59)$ & $45 \%(45)$ \\
\hline Discoidal & $13 \%(10)$ & $46 \%(6)$ & $37 \%(49)$ & $41 \%(41)$ \\
\hline \multicolumn{5}{|l|}{ Tipo Histológico } \\
\hline Adenocarcinoma & $86 \%(66)$ & $85 \%(11)$ & $72 \%(95)$ & $91 \%(91)$ \\
\hline A. mucinoso & $14 \%(11)$ & $15 \%(2)$ & $23 \%(30)$ & $9 \%(9)$ \\
\hline C. de células en anillo de sello & - & - & $5 \%(7)$ & - \\
\hline \multicolumn{5}{|l|}{ Grado de diferenciación } \\
\hline Bien & $9 \%(7)$ & - & $15 \%(20)$ & $12 \%(12)$ \\
\hline Moderado & $70 \%(54)$ & $100 \%(13)$ & $76 \%(100)$ & $76 \%(76)$ \\
\hline Poco & $21 \%(16)$ & - & $9 \%(12)$ & $12 \%(12)$ \\
\hline \multicolumn{5}{|l|}{ Infiltración Tumoral } \\
\hline T1 & $8 \%(6)$ & - & $9 \%(12)$ & $5 \%(5)$ \\
\hline $\mathbf{T} 2$ & $14 \%(11)$ & $23 \%(3)$ & $18 \%(24)$ & $17 \%(17)$ \\
\hline T3 & $71 \%(55)$ & $62 \%(8)$ & $67 \%(88)$ & $73 \%(73)$ \\
\hline T4 & $7 \%(5)$ & $15 \%(2)$ & $6 \%(8)$ & $5 \%(5)$ \\
\hline Linfonodos resecados (promedio) & $19,1 \pm 14$ & $11,1 \pm 8$ & $13,6 \pm 9$ & $11 \pm 7$ \\
\hline $\begin{array}{l}\text { Linfonodos comprometidos } \\
\text { (promedio) }\end{array}$ & $1,1 \pm 2$ & $1,7 \pm 3$ & $1,2 \pm 2$ & $1,2 \pm 3$ \\
\hline
\end{tabular}



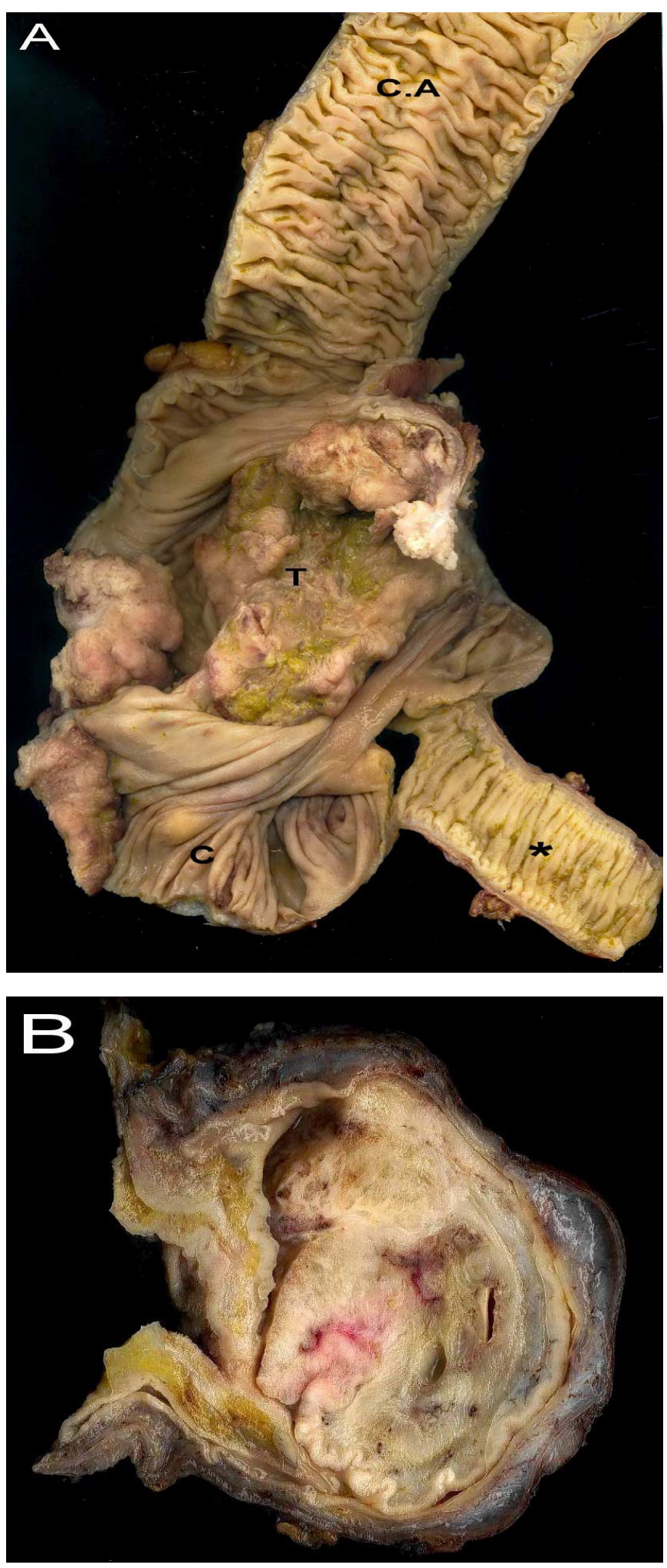

Fig. 1. 1A. Pieza quirúrgica que incluye íleon distal (*), ciego (c) y colon ascendente (C.A); en la cual se reconoce a nivel cecal gran lesión tumoral (T) exofítica de 14 cm de diámetro mayor, apreciándose su crecimiento vegetante en Fig. 1B. tenían diferenciación tubular, papilar y tubulopapilar el $73 \%, 22 \%$ y $5 \%$ respectivamente. Otros tipos histológicos identificados incluyen adenocarcinoma mucinoso (16\%) y carcinoma de células en anillo de sello (2\%).

La distribución de acuerdo al nivel de infiltración tumoral fue T1 7\%, T2 17\%, T3 70\% y T4 6\%.

De los linfonodos examinados el $39 \%$ presentó compromiso tumoral, de estos el $95 \%$ de los casos correspondió a tumores estadio $\mathrm{T} 3$ y $\mathrm{T} 4$. El promedio de linfonodos resecados y con compromiso tumoral fue $14,1 \pm 10,7$ y $1,25 \pm 2,5$ respectivamente.

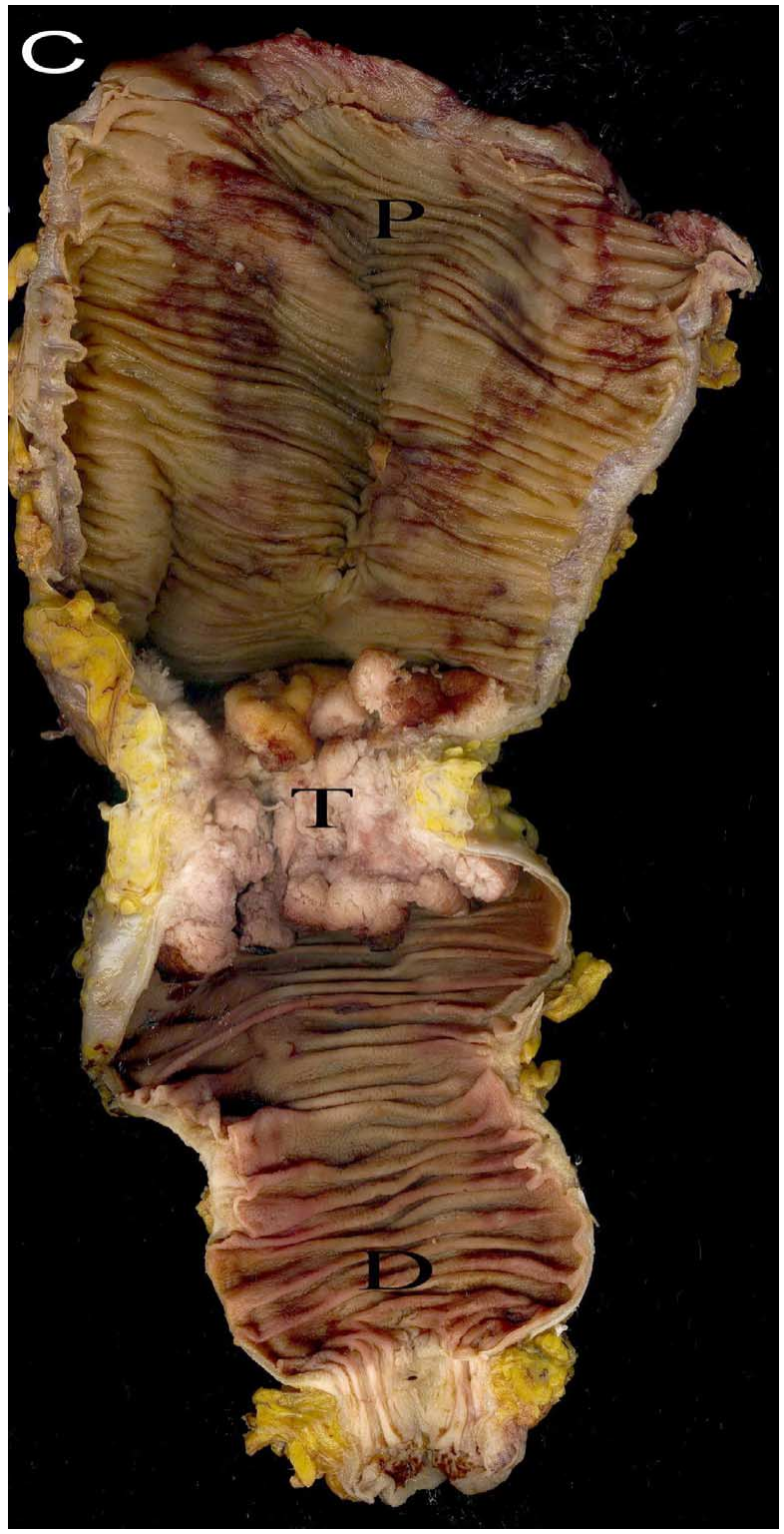

Fig. 2. Pieza quirúrgica de colon sigmoides en la que se observa lesión tumoral $(\mathrm{T})$ de disposición anular y estenosante con dilatación de segmento proximal (P). 


\section{DISCUSIÓN}

En nuestro grupo de estudio, al igual que en otras series publicadas la distribución de los CCR en el intestino grueso son más frecuentes en el colon izquierdo y recto, siendo afectadas generalmente personas mayores de 60 años con una edad promedio de 63,7 años (Zúñiga et al., 2005).

Respecto a la forma tumoral y su relación de acuerdo a la localización en colon derecho o izquierdo, las lesiones exofíticas o polipoideas son más frecuentes en el colon derecho mientras que lesiones de disposición anular y generalmente estenosantes son más frecuentes en colon izquierdo, coincidiendo con lo reportado por otros autores (Speights et al.; Barillari et al.; Qizilbash et al.; Fenoglio). Del mismo modo coincidimos con lo publicado en cuanto los cánceres en colon derecho son más grandes que los de colon izquierdo ( $\mathrm{p}<0,001)$ (Fenoglio; Odze \& Goldblum; Ikeda et al., 1996; Saltztein et al., 1998). A nivel rectal resultó difícil en ocasiones definir la forma tumoral producto de los efectos producidos por el tratamiento neoadyuvante que recibieron algunos pacientes, siendo en el $58 \%$ de los casos lesiones ulceradas en ocasiones pequeñas y con acentuada fibrosis de la pared intestinal.

El tipo histológico más frecuente fue el adenocarcinoma (82\%) ya sea con diferenciación tubular, papilar o tubulopapilar y en $16 \%$ y $2 \%$ adenocarcinoma mucinoso y carcinoma de células en anillo de sello respectivamente (Fenoglio, Odze \& Goldblum; WHO).
A pesar de la existencia de una fase preclínica prolongada y de lesiones precursoras de esta neoplasia que permitirían su detección en etapas precoces con el consiguiente impacto en la supervivencia, observamos en nuestra serie compromiso tumoral de linfonodos en el 39\%, correspondiendo a tumores estadio T3 y T4 el 72\% de los casos; dado su valor pronóstico demostrado en múltiples series, estas variables están incluidas en todas las clasificaciones de estadificación vigentes para los tumores de colon y recto (Jass \& Morson, 1986; Compton \& Greene, 2004; AJCC; Newland et al., 1994). Las clasificaciones actuales para estos tumores considera necesario un mínimo de 12 linfonodos para etapificar un paciente, siendo el promedio resecado en nuestro estudio 14,1 .

En Chile y otros países de Latinoamérica, sin embargo, la realización de pruebas para detección precoz es todavía controversial, entre estos están la prueba de hemorragias ocultas (THO), la sigmoidoscopía y colonoscopía. Puesto que el rendimiento de un método de tamizaje depende de la prevalencia de la patología que se estudia, en nuestro país sería necesario efectuar aproximadamente 1100 THO para prevenir 1 muerte por CCR en 10 años; incluso en EE.UU. y Europa (zonas geográficas de mayor prevalencia e incidencia que Chile) aún existe controversia respecto del costo/beneficio de un programa de detección precoz (Bannura, Kronborg et al., 1996; Bixquert Jiménez, 2006).

TAPIA, E. O.; ROA, S. J. C.; MANTEROLA, D. C. \& BELLOLIO, J. E. Colon and rectum cancer: morphological and clinical description of 322 cases. Int. J. Morphol., 28(2):393-398, 2010.

SUMMARY: The colorectal cancer (CRC) is currently the fourth cause of cancer death in Chile. Its incidence, however, is continuously increasing in our population. The aim of this study is to describe morphological and clinical aspects of patients resected CRC. Retrospective cohort study. We studied 322 patients operated on for CRC between 1987 and 2003 in the Hernan Henriquez Aravena Hospital of Temuco. The clinical and morphological variables studied (all analyzed for subgroups of subjects with tumors of the colon and rectum) were age, gender, tumor location, tumor size and shape, level of infiltration, histological type, histological differentiation grade and tumor involvement lymph node. We used descriptive statistics and analytical, using Pearson chi-square and Fisher exact tests for categorical variables and T-test for continuous variables. The median age was66 years, with average age of the right colon tumors $(\mathrm{RC})$, transverse (TC), left $(\mathrm{LC})$ and rectum was 62.2, 64.6, 64, and 64.4 years respectively $(\mathrm{p}=0.53)$. In $\mathrm{RC}$ and LC were observed $57 \%$ and $47 \%$ of women, respectively ( $\mathrm{p}<0.05) .69 \%$ of the cases corresponded to tumors of the colon $(24 \% \mathrm{RC}$, $4 \% \mathrm{TC}$ and $41 \% \mathrm{LC}$ ) and $31 \%$ to cancer of the rectum. The average tumor size was $67.2 \pm 33.1 \mathrm{~mm} \mathrm{RC,} 53.5 \pm 19.7 \mathrm{~mm}$. TC, $44.1 \pm$ $22.3 \mathrm{~mm} \mathrm{LC}$ and $41.5 \pm 17.5 \mathrm{~mm}$ in the rectum ( $\mathrm{p}<0.001$ ). On RC as Bormann type I was observed in $57 \%$ while the LC annular and ulcerative lesions $45 \%$ CI. We found $75 \%$ of moderately differentiated tumors, corresponding to $82 \%$ adenocarcinomas, $16 \%$ and $2 \%$ mucinous adenocarcinoma cell carcinoma signet-ring. 76\% were T3 and T4 tumors. We found lymph node tumor involvement in 39\% of which $95 \%$ were T3-T4 tumors. We checked at the regional level for patients with CRC clinical and morphological variables described in the literature.

KEY WORDS: Colorectal cancer; Colon cancer; Rectal cancer. 


\section{REFERENCIAS BIBLIOGRÁFICAS}

AJCC (American Joint Committee on Cancer). Colon and rectum. AJCC Cancer Staging Manual. $6^{\text {th }}$ ed. New York, Springer, 2002. pp.113-24.

Bannura, G. ¿Se puede mejorar la oportunidad del diagnóstico del cáncer colorrectal en Chile? Rev. Chil. Cir., 58:59-61, 2006.

Bannura, G.; Contreras, J.; Cumsille, M. A.; García, C. \& Portalier, P. Results of the surgical treatment of colorectal cancer: analysis of recurrence and survival in 400 patients. Rev. Med. Chil., 123(4):464-72, 1995.

Bannura, G.; Cumsille, M. A.; Contreras, J.; Melo, C.; Barrera, A.; Reinero, M. \& Pardo, L. Prognostic factors in colorectal neoplasm. Multivariate analysis in 224 patients. Rev. Med. Chil., 129(3):237-46, 2001.

Barillari, P.; de Angelis, R.; Valabrega, S.; Indinnimeo, M.; Gozzo, P.; Ramacciato, G. \& Fegiz, G. Relationship of symptom duration and survival in patients with colorectal carcinoma. Eur. J. Surg. Oncol., 15(5):441-5, 1989.

Bixquert Jiménez, M. Early diagnosis of colorectal cancer. Diagnostic delay reduction or rather screening programs? Rev. Esp. Enferm. Dig., 98(5):315-21, 2006.

Compton, C. C. \& Greene, F. L. The staging of colorectal cancer: 2004 and beyond. C. A. Cancer J. Clin., 54(6):295-308, 2004.

Donoso, A.; Villarroel, L. \& Pinedo, G. Increase in colon cancer mortality rates in Chile, during the period 1990-2003. Rev. Med. Chil., 134(2):152-8, 2006.

Fenoglio, C. Gastrointestinal pathology. $3^{\circ}$ Ed. Philadelphia, Lippincott Williams \& Wilkins, 2008.

Ikeda, Y.; Koyanagi, N.; Mori, M.; Ezaki, T.; Toyomasu, T.; Minagawa, S.; Tateishi, H. \& Sugimachi, K. Increased incidence of proximal colon cancer in the elderly. J. Clin. Gastroenterol., 23(2):105-8, 1996.

Jass, J. R. \& Morson, B. C. A universal staying system of cancer of de colon and rectum revised. Arch. Pathol. Lab. Med., 110:1119, 1986.

Kronborg, O.; Fenger, C.; Olsen, J.; Jørgensen, O. D. \& Søndergaard, O. Randomised study of screening for colorectal cancer with faecal-occult-blood test. Lancet, 348(9040):146771, 1996.

Landis, S. H.; Murray, T.; Bolden, S. \& Wingo, P. A. Cancer statistics, 1999. C. A. Cancer J. Clin., 49(1):8-31, 1, 1999.

López, F. P. G.; Zárate, A.; Avendaño, R. \& Soto, G. Utilidad de la endosonografía rectal en la etapificación preoperatoria del cáncer de recto. Rev. Chil. Cir., 55:136-40, 2003.

Newland, R. C.; Dent, O. F.; Lyttle, M. N.; Chapuis, P. H. \& Bokey, E. L. Pathologic determinants of survival associated with colorectal cancer with lymph node metastases. A multivariate analysis of 579 patients. Cancer, 73(8):2076-82, 1994.

Odze, R. \& Goldblum, J. Surgical pathology of the GI tract, liver, biliary tract and pancreas. $2^{\circ}$ Ed. Philadelphia, Elsevier, 2009.

Parkin, D. M.; Muir, C. S.; Whelan, S. L.; Gao, Y. T.; Ferlay, J. \& Powell, J. Cancer inidence in five continents. 6(120), Lyon, IARC scientific publication, 1992.

Qizilbash, A. H. Pathologic studies in colorectal cancer. A guide to the surgical pathology examination of colorectal specimens and review of features of prognostic significance. Pathol. Аппи., 17(1):1-46, 1982.

Saltzstein, S. L.; Behling, C. A. \& Savides, T. J. The relation of age, race, and gender to the subsite location of colorectal carcinoma. Cancer, 82(7):1408-10, 1998.

Speights, V. O.; Johnson, M. W.; Stoltenberg, P. H.; Rappaport, E. S.; Helbert, B. \& Riggs, M. Colorectal cancer: current trends in initial clinical manifestations. South Med. J., 84(5):575-8, 1991.

WHO. World Health Organization Classification of Tumours. Pathology and Genetics of Tumours of the Digestive System. Lyon, France, IARC scientific publication, 2000.

Ziegler, R. C.; Revesa, S. S. \& Fraumeni, J. F. Epidemiologic patterns of colorectal cancer. De Vita, V. T. Jr.; Hellman, S. \& Rosenberg, S. A. (Eds). Important advances in oncology. Philadelphia, JB Lippincott, 1986. p.209.

Zúñiga, A. L. F.; Boza, C.; Zúñiga, A. \& Rahmer, A. Análisis uni y multivariado de los factores pronósticos en cáncer de colon. Rev. Chil. Cir.; 57(2):143-8, 2005.

Dirección para correspondencia:

Dr. Oscar Tapia E.

Departamento de Anatomía Patológica

Facultad de Medicina

Universidad de La Frontera.

Manuel Montt 112

Código Postal 478-1176

Temuco - CHILE

Email: jcroa@ufro.cl

Recibido : 17-12-2009

Aceptado: 15-03-2010 